\title{
Pubic Symphysitis: A Little-Known Complication of Radical Prostatectomy
}

Khdach Youness $^{1 *}$, Hamedoun Larbi ${ }^{1 *}$, Boukhlifi Youness ${ }^{1}$, Alami Mohamed $^{1}$, Ameur Ahmed $^{1}$

\footnotetext{
${ }^{1}$ Service of Urology, Military Hospital of Instruction Mohamed V, Hay Ryad - 10100 RABAT, Morocco
}

DOI: $\underline{10.36347 / \mathrm{sasjs} .2021 . v 07 i 02.016}$

| Received: 07.02.2021 | Accepted: 19.02.2021 | Published: 21.02.2021

*Corresponding author: Khdach Youness

Abstract

Case Report

Post-surgery pubic symphysis is a rare condition that is difficult to diagnose. It must be mentioned in front of any pubic pain associated with a fever. The biological assessment is often disturbing but not specific. The MRI most often allows the diagnosis to be made and guides the sample to be taken which allows the antibiotic therapy to be adapted. The treatment is based on a long-term combination of antibiotics, the evolution is most often favourable. We report here the case of pubic symphysitis complicating a radical prostatectomy in our centre.

Keywords: Pubic sympositis, radical prostatectomy, urinary tract infection, MRI, puncture biopsy.

Copyright $\left({ }_{0} 2021\right.$ The Author(s): This is an open-access article distributed under the terms of the Creative Commons Attribution 4.0 International License (CC BY-NC 4.0) which permits unrestricted use, distribution, and reproduction in any medium for non-commercial use provided the original author and source are credited.

\section{INTRODUCTION}

Infectious pubic symphysitis is a rare condition that can complicate any type of urological [1] or gynaecological surgery, first described by Ellioston in 1827 and then Legueu and Roche in 1923 [2]. It is a septic arthritis often unknown, with deceptive symptoms suspected in front of pain in the pelvic region associated with a fever, and with an incidence difficult to establish in the general population, all the more so as the nosology remains unclear in the literature [3] as well as other etiologies have been described [4]. Here we report the case of a pubic symphisitis complicating a radical prostatectomy in our training.

\section{CLINICAL OBSERVATION}

A 60-year-old patient, hypertensive on amlodipine and diabetic on metformin, admitted to our department for the management of an adenocarcinoma of the prostate Gleason 7(4+3) ISUP 2, classified T2a NOM0 (Figure-1), the patient underwent a laparoscopic radical prostatectomy in June 2020 (Figure-2). The immediate post-operative follow-up was simple and the patient was declared out after 03 days but secondarily he returned to the clinic with inflammatory pubic pain, responding very poorly to non-steroidal antiinflammatory drugs, associated with significant functional impotence. In view of this clinical picture, the patient benefited from a pelvic MRI scan which revealed the presence of a disjunction of the pubic symphysis associated with intra-articular fluid effusion and oedematous infiltration of the ischio and ilio-pubic branches (Figure-3). After hospitalisation of the patient, clinical examination found a febricula at $38^{\circ} \mathrm{C}$, the patient complained of bilateral inguinal pain and palpation revealed a sensitivity and arching of the pubic symphysis (Figure-4). A biological assessment carried out shows an infectious syndrome with a CRP of 106 $\mathrm{mg} / \mathrm{l}$ and hyperleukocytosis at 5487, the PSA was 0.11 $\mathrm{ng} / \mathrm{ml}$. Bacteriological examination of urine found a Pseudomonas aerogenosa infection. A scan-guided biopsy of the pubic symphysis was then carried out and revealed two germs: Staphylococcus aureus and Pseudomonas aeruginosa (Figure-5).

The patient received IV bi-antibiotic therapy combining ceftazidine $3 \mathrm{~g} /$ day and ciprofloxacin 400 $\mathrm{mg} /$ day for 14 days, followed by an oral relay for 6 weeks.

The evolution was marked by the disappearance of pain and functional impotence, CRP negativation and urine sterilisation.

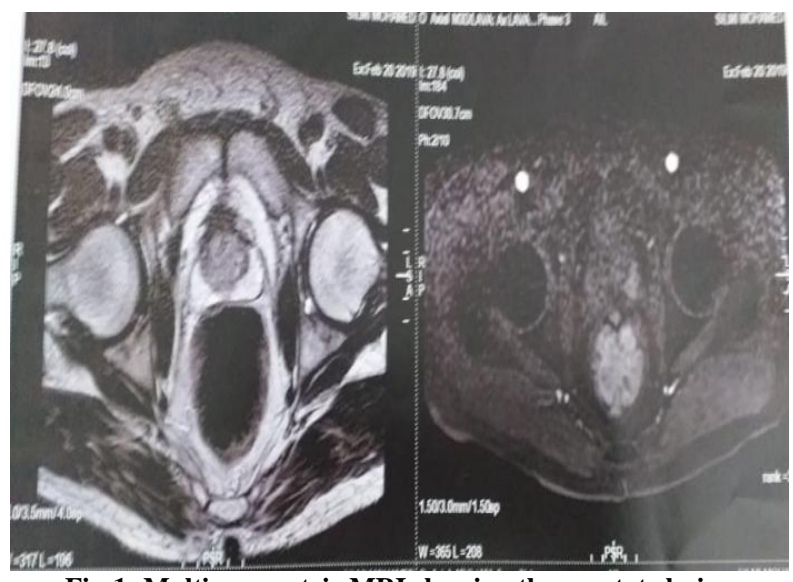

Fig-1: Multiparametric MRI showing the prostate lesion 
Khdach youness et al., SAS J Surg, Feb, 2021; 7(2): 93-95

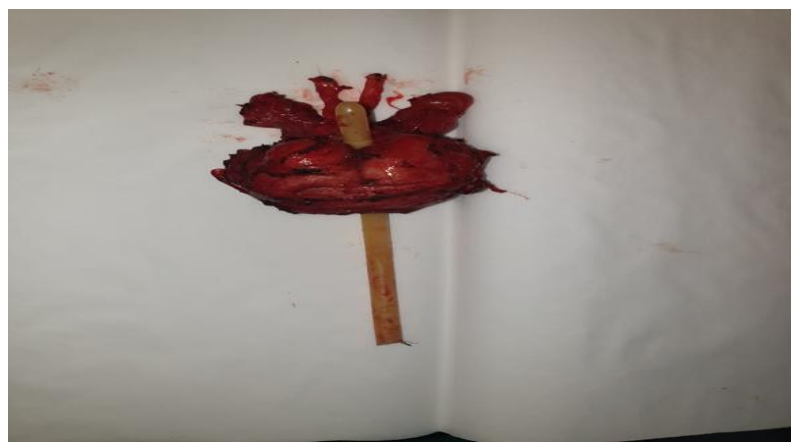

Fig-2: The surgical part of the laparoscopic radical prostatectomy

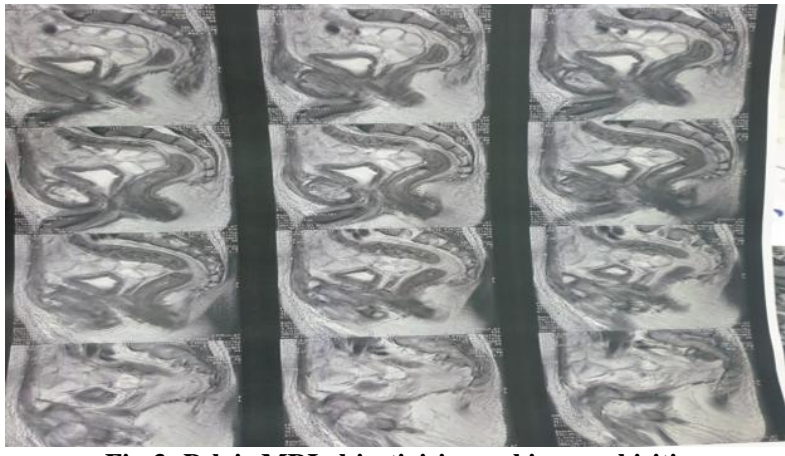

Fig-3: Pelvic MRI objectivising pubic symphisitis

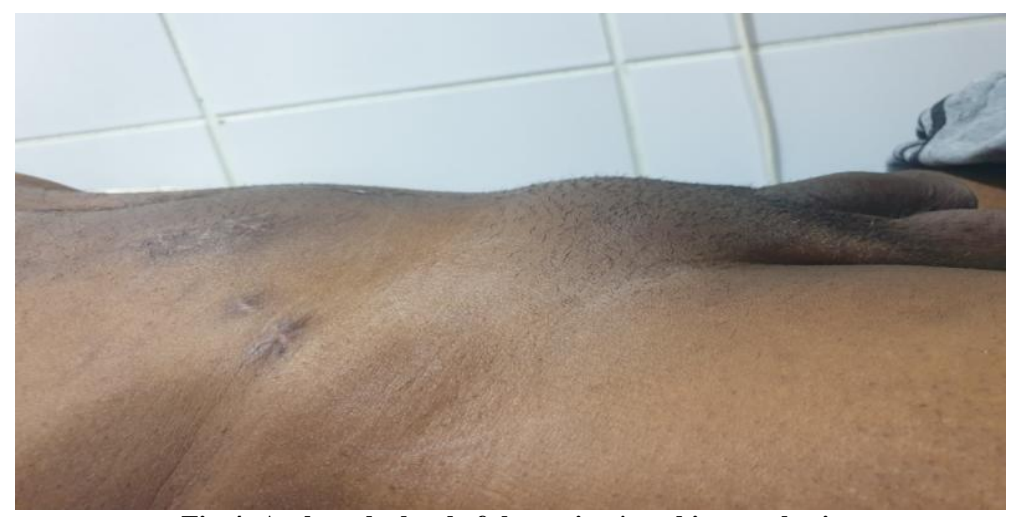

Fig-4: Arch at the level of the patient's pubic symphysis

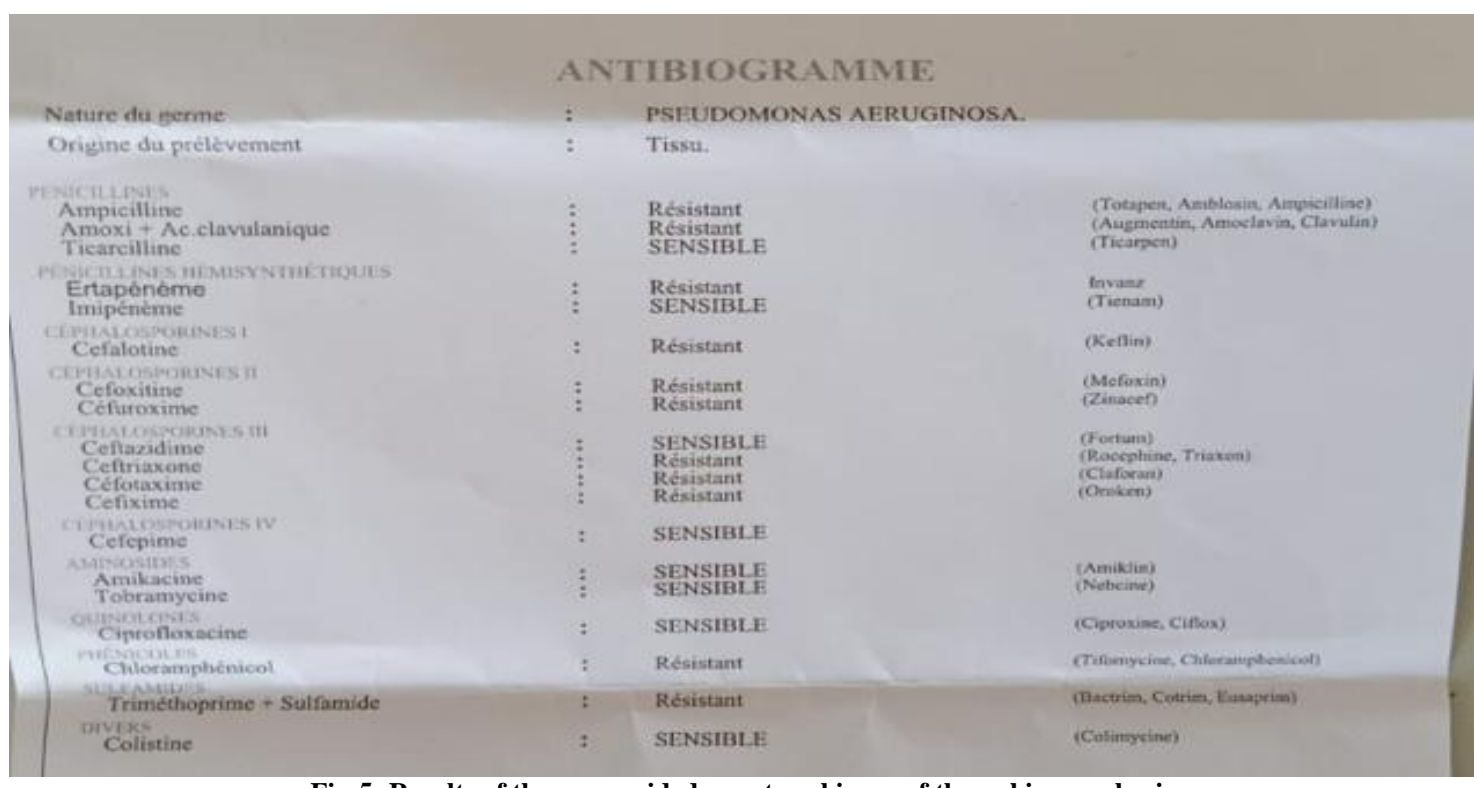

Fig-5: Results of the scan-guided puncture biopsy of the pubic symphysis

\section{DISCUSSION}

Pubic symphysis, a rare complication, often occurs in a field with favourable conditions, namely: any uro-gynecological surgery with close contact with the pubis (radical prostatectomy as in our case, prostate biopsy, bladder catheterisation, cystectomy, incontinence treatment, childbirth, abortion [5, 6] associated with urethral or bladder perforation, scar or urinary infection as in our case.

According to the latest recommendations of the Infectious Diseases Committee of the French
Urological Society [7], no antibiotic prophylaxis is recommended for radical prostatectomy [2].

According to various authors, the first symptoms appear 2 weeks to 3 months after the causal operation $[2,6,8,7]$. This often misleading clinical symptomatology is often misunderstood and is manifested by the appearance of exquisite pain at the pressure of the pubis and fever, biologically inconsistent hyperleukocytosis (27 and $20 \%$ of cases) [5], high SV and CRP [6]. 
The standard radiograph of the pelvis can be interesting by showing a widening of the joint space with blurred edges and erosions in 2 to 4 weeks. Osteocondensation and periosteal appositions sometimes leading to synostosis are late signs of appearance.

The CT scan is a more precise and sensitive examination of pubic symphysitis, and allows a better visualization of soft tissue invasion, bone sequestration, abscess or possible complications and to guide a sampling.

MRI allows early detection of oedema and inflammation of the bone and muscles, intra-articular effusion or abscess [9].

Then you have to look for the responsible germ. This germ can be isolated in blood cultures or lochies in postpartum. If this investigation is negative, a local sampling by trocar biopsy or surgical scano or echo-guided sampling may be proposed. It should be noted that although urine cultures are sometimes positive, the germ identified may be different from the germ found at the puncture-biopsy of the pubic symphysis. Direct sampling of the pubic symphysis should therefore be carried out if there is any doubt. Moreover, the low virulence of these germs, their long culture time, or prior antibiotic therapy, may lead to the culture being wrongly considered sterile.

The most frequently incriminated germs are: the staphylococcus of a Pseudomonas aeruginosa of Escherichia coli, a Proteus mirabilis after urological surgery, post-partum streptococcus B and anaerobic and polymicrobial infections other germs can be found: pneumococcus, brucella, Haemophilus paraphrophilus, enterococcus faecalis, salmonella Indiana, peptostreptococcus, mycobacterium tuberculosis [7].

The treatment is based on a combination of 2 IV antibiotics initially for the first two weeks followed, if the evolution is favourable, by a per os relay. The total duration of the treatment is 6 weeks for the majority of authors, or even several weeks depending on the clinical and biological evolution. In case of failure of the medical treatment or in case of sequestration, intra bony foreign body, or abscess, surgical curettage is indicated [10].

For our patient the evolution was very satisfactory after 2 weeks of targeted antibiotic therapy with a sterile echo-guided puncture biopsy, which gave way for the per os relay to total 6 weeks of treatment.

\section{CONCLUSION}

A rare complication of urological surgery, it is an infectious disease that is difficult to diagnose. It must be suspected at the slightest post-operative pubic pain associated with a fever. MRI and pubic puncture biopsy are of considerable help in diagnosis and therapeutic choice. The treatment is based on an adapted and prolonged antibiotic therapy.

Conflicts of Interest: The authors do not declare any conflict of interest.

Authors' Contributions: Khdach Youness and Hamedoun LARBI are the main authors. Boukhlifi youness is the second authors. Alami Mohamed is the head of the urology department. Ahmed Ameur is the head of the urological surgery pole who oversaw patient care and led the project.

\section{REFERENCES}

1. Burns JR, Gregory JG. Osteomyelitis of the pubic symphysis after urologic surgery. J Urol. 1977;118:803-5.

2. Salomon S, Lasselin-Boyard P, Lasselin J, Goëb V. Symphysite pubienne infectieuse postchirurgicale. Progrès en urologie. 2015 Mar 1;25(3):169-74.

3. Arlet J, Bouteiller G, Durroux R, Sebbag D, SaintPrix C, Arlet P, Naudon J. Pubic and ischio-pubic osteitis. Bacteriological and histopathological study of the pubic bone. Revue du rhumatisme et des maladies osteo-articulaires. 1981 Feb 1;48(2):101-6.

4. Ross JJ, Hu LT. Septic arthritis of the pubic symphysis: review of 100 cases. Medicine. 2003; 82:340-5.

5. Charles P, Ackermann F, Brousse C, Piette AM, Blétry O, Kahn JE. Spontaneous streptococcal arthritis of the pubic symphysis. La Revue de medecine interne. 2010 Sep 20;32(7):e88-90.

6. Kats E, Venema PL, Kropman RF, Kieft GJ. Diagnosis and treatment of osteitis pubis caused by a prostate-symphysis fistula: a rare complication after transurethral resection of the prostate. British journal of urology (Print). 1998;81(6):927-8.

7. Almeras C, Madi F, Desplaces N, Mamoudy P. Postoperative pubic osteitis: diagnostic and therapeutic management and results. Prog Urol. 2002; 12:253-9.

8. Andonian S, Rabah DM, Aprikian AG. Pseudomonas aeruginosa sacroiliitis and osteomyelitis of pelvic bones after radical prostatectomy. Urology. 2002; 60:698.

9. Jarlaud T, Railhac JJ, Sans N, De Paulis F. Normal and pathologic symphysis pubis: value of imaging. J Radiol. 2001; 82:425-36.

10. Grace JN, Sim FH, Shives TC, Coventry MB. Wedge resection of the symphysis pubis for the treatment of osteitis pubis. J BoneJoint Surg. 1989;71:3586-94. 